\title{
Core plasma ion cyclotron emission driven by fusion-born ions
}

Ochoukov, R.; Bilato, R.; Bobkov, V.; Chapman, B.; Chapman, S.C.; Dendy, R.O.; Dunne, M.; Faugel, H.; García-Muñoz, M.; Geiger, B.

Total number of authors:

19

Published in:

Nuclear Fusion

Link to article, DOI:

10.1088/1741-4326/aaebb0

Publication date:

2019

Document Version

Peer reviewed version

Link back to DTU Orbit

Citation (APA):

Ochoukov, R., Bilato, R., Bobkov, V., Chapman, B., Chapman, S. C., Dendy, R. O., Dunne, M., Faugel, H., García-Muñoz, M., Geiger, B., Kallenbach, A., Kappatou, A., McClements, K. G., Moseev, D., Nielsen, S., Rasmussen, J., Schneider, P., Weiland, M., \& Noterdaeme, J-M. (2019). Core plasma ion cyclotron emission driven by fusion-born ions. Nuclear Fusion, 59(1), [014001]. https://doi.org/10.1088/1741-4326/aaebb0

\section{General rights}

Copyright and moral rights for the publications made accessible in the public portal are retained by the authors and/or other copyright owners and it is a condition of accessing publications that users recognise and abide by the legal requirements associated with these rights.

- Users may download and print one copy of any publication from the public portal for the purpose of private study or research.

- You may not further distribute the material or use it for any profit-making activity or commercial gain

- You may freely distribute the URL identifying the publication in the public portal 


\title{
Core plasma ion cyclotron emission driven by fusion-born ions
}

R. Ochoukov, ${ }^{1}$ R. Bilato, ${ }^{1}$ V. Bobkov, ${ }^{1}$ B. Chapman, ${ }^{2}$ S.C. Chapman, ${ }^{2}$ R.O. Dendy, ${ }^{2,3}$ M. Dunne, ${ }^{1}$ H. Faugel, ${ }^{1}$ M. García-Muñoz, ${ }^{4}$ B. Geiger, ${ }^{5}$ A. Kallenbach, ${ }^{1}$ A. Kappatou, ${ }^{1}$ K. G. McClements, ${ }^{3}$ D. Moseev, ${ }^{5}$ S. Nielsen, ${ }^{6}$ J. Rasmussen, ${ }^{6}$ P. Schneider, ${ }^{1}$ M. Weiland, ${ }^{1}$ J.-M. Noterdaeme, ${ }^{1,7}$ ASDEX Upgrade Team, ${ }^{\text {a) }}$ and EUROfusion MST1 Team ${ }^{\text {b) }}$

${ }^{1}$ Max Planck Institute for Plasma Physics, Boltzmannstr. 2, D-85748 Garching, Germany ${ }^{2}$ Centre for Fusion, Space and Astrophysics, University of Warwick, Coventry, CV4 7AL, United Kingdom ${ }^{3} \mathrm{CCFE}$, Culham Science Centre, Abingdon, Oxfordshire, OX14 3DB, UK ${ }^{4}$ FAMN Department, Faculty of Physics, University of Seville, 41012 Seville, Spain ${ }^{5}$ Max Planck Institute for Plasma Physics, Wendelsteinstr. 1, 17491, Greifswald, Germany ${ }^{6}$ Department of Physics, Technical University of Denmark, Fysikvej, b. 309, DK-2800 Kgs. Lyngby, Denmark ${ }^{7}$ Applied Physics Department, UGent, 9000 Gent, Belgium

a) see the author list in A. Kallenbach et al., Nucl. Fusion 57, 102015 (2017).

b) see the author list in H. Meyer et al., Nucl. Fusion 57, 102014 (2017).

\begin{abstract}
Ion cyclotron emission (ICE) signals whose spectral peaks match the fundamental cyclotron frequencies of hydrogen and tritium in the plasma core, near the magnetic axis, are observed in ASDEX Upgrade deuterium plasmas. In these cases the only source of energetic $(\geq 1 \mathrm{MeV})$ hydrogen and tritium ions is D-D fusion reactions between neutral beam injected deuterium ions and bulk deuterium ions. Hydrogen-matched core ICE is observed in a wide variety of ASDEX Upgrade plasmas, while tritium-matched core ICE is (so far) only observed in socalled H-mode density limit plasmas. In all cases ICE signals are detected directly using B-dot probes, which provide information on the emission frequency, the amplitude, and, in principle, the parallel wavenumber values. These observations support the idea of using an ICE-based diagnostic to monitor the presence of fusion-born alpha particles in tritium-burning fusion plasmas on devices such as JET, ITER, CFETR, or DEMO.
\end{abstract}

\section{Main Paper}

We present experimental evidence for fusion product-driven ion cyclotron emission (ICE) from the core region of ASDEX Upgrade tokamak plasmas. The most common spectral peak in the core ICE signal measured on ASDEX Upgrade has a frequency that matches the fundamental hydrogen cyclotron frequency at the plasma center [1]. Accelerated thermalized minority hydrogen ions can be excluded in these cases, as there is no ion cyclotron range-of frequencies (ICRF) power applied to accelerate the ions. In fact, the source of energetic (a.k.a. fast) hydrogen in these plasmas is one of the two possible D-D fusion reactions: $\mathrm{D}+\mathrm{D} \rightarrow \mathrm{T}+$ $\mathrm{H}$ (with $\mathrm{D}+\mathrm{D} \rightarrow{ }^{3} \mathrm{He}+$ neutron being the second possible reaction). However, this hydrogen frequency coincides with the second cyclotron harmonic of deuterium, and energetic deuterium ions are also present in these plasmas through neutral beam injection (NBI). We now complement these results with ICE observations whose frequencies match the fundamental tritium cyclotron frequency in the plasma center. These ICE signals have been observed for the first time on ASDEX Upgrade during high density H-mode plasmas. The source of fast tritium is, again, D-D fusion reactions. This result is important as it strengthens the case of an ICE-based passive non-intrusive diagnostic to monitor the presence of fusionborn alpha particles in D-T magnetized fusion plasmas in JET, ITER, CFETR, or DEMO [2].

ICE is an electro-magnetic wave excited in toroidal magnetically confined plasmas by the collective instability of suprathermal ions [3]. The emission frequency of the fundamental 
harmonic is in the ICRF range of $10-100 \mathrm{MHz}$ on conventional tokamaks or stellarators. ICE is typically detected at discrete, regularly spaced frequencies, which enables one to determine (or match) the radial position of its origin via the cyclotron relation: $\omega_{c i}=q_{i} B / m_{i}$, where $\omega_{\mathrm{ci}}$ is the angular cyclotron frequency of the driving ion species $\mathrm{i}, \mathrm{q}_{\mathrm{i}}$ is the ion charge, $B$ is the local magnetic field strength, and $m_{i}$ is the ion mass. ICE originating from the lowfield side plasma edge is by far the most common type (see [2] and references therein). The fast ions driving the instability can be introduced directly via energetic neutral beam injection (NBI) [4], accelerated by externally-launched ICRF waves [5], or produced via nuclear fusion reactions [6]. More recently, core ICE observations have been reported from three tokamaks: ASDEX Upgrade [1], DIII-D [7], and TUMAN-3M [8]. In these cases, deuterium plasmas heated with deuterium NBI show ICE at the fundamental hydrogen $\omega_{\mathrm{CH}}$ (and the second harmonic deuterium $\omega_{\mathrm{CD}}$ ) cyclotron frequency near the plasma center, not the low-field side edge where ICE is more commonly observed [2]. These core emission signals have been measured with detectors placed on both the low- and the high-field sides of these tokamaks, implying that the excited waves can traverse the confined plasma region without significant scattering or reabsorption. Importantly, these observations raise the possibility of using such waves to measure properties of the fusion-born ion population in the plasma core.

For the case of ASDEX Upgrade, ICE is detected directly using magnetic induction coils (or B-dot probes) placed in the far periphery of the machine, essentially in the vacuum region of the tokamak [9]. The radio frequency (RF) magnetic field component sampled by the B-dot probes is then processed via so-called fast and slow data acquisition systems [1]. The fast system digitizes the signal directly at $125 \mathrm{MHz}$ over a $1 \mathrm{~ms}$ (rectangular) time window, every $10 \mathrm{~ms}$, for a duration of 8 seconds, which is long enough for most ASDEX Upgrade plasmas [1]. The slow system first rectifies the RF signal from the B-dot probes and then digitizes the resulting DC output continuously at $200 \mathrm{kHz}$ [1]. The slow system can accept two RF signals simultaneously to measure the phase difference $\phi$ between them [1]. To measure the phase (and, hence, the parallel wavenumber $k / /$ ), a pair of B-dot probes is positioned in the periphery of the ASDEX Upgrade torus radially $10 \mathrm{~cm}$ behind the limiter surface, on the low field side [1]. The probes are spaced $2.4 \mathrm{~cm}$ apart, along the direction of a typical local magnetic field line (on-axis magnetic field $\mathrm{B}_{\mathrm{T}}=2.50 \mathrm{~T}$, plasma current $\mathrm{I}_{\mathrm{P}}=1.0 \mathrm{MA}$ ). The orientation of the inductors is parallel to the typical local magnetic field line meaning that the probes preferentially detect fast ICRF waves.

A core ICE signal with frequency matching $\omega_{\mathrm{CH}}$ (and degenerate with $2 \times \omega_{\mathrm{CD}}$ ) is often observed in ASDEX Upgrade deuterium plasmas heated with deuterium NBI [1]. An example is provided in figure 1, where a train of NBI pulses (figure 1 (c)) triggers an instability pulse train at 39-40 MHz (figure 1 (a)). Once developed, the ICE in figure 1 (a) remains in phase with the NBI turn-on time and the measured frequency matches $\omega_{\mathrm{CH}}$ (and $2 \times \omega_{\mathrm{CD}}$ ) at the magnetic center: $\mathrm{f} \sim 39.8 \mathrm{MHz}$ where $\mathrm{B}_{\mathrm{T}}=2.62 \mathrm{~T}$ (figure 1 (a)). As the NBI power blips modulate the plasma pressure and, hence, the radial position of the magnetic axis (figure 1 (b)), the measured ICE frequency follows this modulation (figure 1 (a)). Since $\omega_{\mathrm{CH}}$ and $2 \times \omega_{\mathrm{CD}}$ coincide, it is not possible to unambiguously determine from this observation alone, which fast ion species is driving the emission in figure 1. Both species are present in ASDEX Upgrade deuterium plasmas heated with deuterium NBI: the fast hydrogen is produced via D$\mathrm{D}$ fusion (with birth energy $\mathrm{E}_{\mathrm{o}}=3.02 \mathrm{MeV}$ ) and the fast deuterium is injected directly by the NBI system $\left(E_{o}=50-60 \mathrm{keV}\right.$ in this particular discharge). For this reason, detection of core ICE due to a different D-D fusion product, either tritium $\left(E_{o}=1.01 \mathrm{MeV}\right)$ or helium-3 $\left(\mathrm{E}_{\mathrm{o}}=\right.$ 
$0.82 \mathrm{MeV}$ ) lends support to the fusion product origin hypothesis. In this manuscript, we present the first observation of ICE with frequency matching $\omega_{\mathrm{CT}}$ at the plasma center: $\mathrm{f} \sim 12.0$ $\mathrm{MHz}$ for $\mathrm{B}_{\mathrm{T}}=2.37 \mathrm{~T}$.

We typically observe ICE in the 10-12 MHz range in so-called H-mode density limit (HDL) plasmas in ASDEX Upgrade [10], see for example figure 2. HDL plasmas are characterized by a strong ramp of $\mathrm{P}_{\mathrm{NBI}}$ (figure 2 (c)). During this ramp, two distinct ICE signals often appear, one in the frequency range $10-11 \mathrm{MHz}$ and the second at $\sim 12 \mathrm{MHz}$ (figure 2 (a)). The first signal is identified as emission at $\omega_{\mathrm{C}}$ near the plasma edge, specifically at the poloidal flux surface location $\rho_{\text {pol }} \sim 0.8$ or, equivalently, at the major radius $\mathrm{R}=2.04 \mathrm{~m}$ on the midplane where $\mathrm{B}=2.05 \mathrm{~T}$. The second signal matches $\omega_{\mathrm{CT}}$ at the plasma center, $\mathrm{R}=1.73 \mathrm{~m}$ where $\mathrm{B}_{\mathrm{T}}=2.37 \mathrm{~T}$, indicated by a dashed red line in figure 2 (a). Both emission types shortlived, lasting for 0.2-0.4 ms at a time, so that the fast ICE diagnostic (which only acquires data for $1 \mathrm{~ms}$, out of every $10 \mathrm{~ms}$ time window) does not capture all of these events (figure 3 (a)). An examination of the measured phase values reveals a large difference between the two signal groups (figure 3 (c)), which helps distinguish core from edge tritium ICE events in the absence of fast ICE diagnostic data (figure 3 (b) and (c)). Note that for display purpose only, each fast data acquisition burst in figure 3 (a) has been expanded from 1 to $10 \mathrm{~ms}$. The core ICE event at $\mathrm{t}=2.70 \mathrm{~s}$ (figure 3 (a)) is captured in its entirety by the fast ICE system (figure 4), and this data reveals a non-trivial behavior of this core ICE type: as the emission frequency value changes, it does so in discrete steps (figure 4 (a)). This "frequency splitting" effect of the core ICE spectrum has been observed on other tokamaks [8], and appears to be a fundamental feature of core ICE.

Our observations provide several key constraints for future first principles models intended to quantify core ICE behavior. First, core ICE that is matched to $\omega_{\mathrm{CH}}$ (and $2 \times \omega_{\mathrm{CD}}$ ) cyclotron frequency is observed in nearly all deuterium discharges heated with deuterium NBI, both Lmode and H-mode alike. This emission generally arises within the first $100 \mathrm{~ms}$ of the NBI turn-on phase before disappearing, but can occasionally last for much longer, $>1 \mathrm{~s}$ [1]. The brief ICE duration during NBI turn-on is consistent with the theoretical expectations that the initially inverted fusion proton velocity distribution takes a finite time (of the order of a collisional slowing-down time) to become monotonically decreasing and, hence, stable [11]. Fusion-born protons $\left(E_{o}=3.02 \mathrm{MeV}\right.$ and birth speed $\left.\mathrm{v}_{\mathrm{o}}=2.41 \times 10^{7} \mathrm{~m} \mathrm{~s}^{-1}\right)$ are partially confined [6] and super-Alfvénic under all plasma conditions on ASDEX Upgrade, unlike NBI deuterium ions $\left(E_{o}=50-90 \mathrm{keV}, \mathrm{v}_{\mathrm{o}}=2-3 \times 10^{6} \mathrm{~m} \mathrm{~s}^{-1}\right)$, whose initial speed is typically a factor of $>2$ below the local Alfvén speed $\mathrm{v}_{\mathrm{A}}=6 \times 10^{6} \mathrm{~m} \mathrm{~s}^{-1}$ in the plasma core where $n_{\mathrm{e}}=4 \times 10^{19} \mathrm{~m}^{-}$ 3. The initial speed of fusion-born tritium ions, on the other hand, $\left(E_{o}=1.01 \mathrm{MeV}, v_{0}=8 \times 10^{6}\right.$ $\mathrm{m} \mathrm{s}^{-1}$ ) is comparable to typical core $\mathrm{v}_{\mathrm{A}}$. However, $\mathrm{v}_{\mathrm{A}}$ value in HDL discharges (where core $\mathrm{n}_{\mathrm{e}}$ reaches $8 \times 10^{19} \mathrm{~m}^{-3}$ ) drops to $4 \times 10^{6} \mathrm{~m} \mathrm{~s}^{-1}$, since $\mathrm{v}_{\mathrm{A}} \sim \mathrm{n}_{\mathrm{e}}{ }^{-1 / 2}$. This makes fusion-born tritium ions super-Alfvénic in HDL ASDEX Upgrade plasmas, which, in turn, makes ICE much more readily driven [12]. We note that, in principle, helium-3 fusion ions could also account for the ICE signal that we identify with tritium ions in Fig. 3, via helium-3 ions with a +1 charge state. However, the large energy difference between fusion-born helium-3 ions $\left(\mathrm{E}_{0}=0.82\right.$ $\mathrm{MeV}$ ) and a possible electron donor (NBI atoms with $\mathrm{E}_{\mathrm{o}}=50-90 \mathrm{keV}$ in these discharges) makes the helium charge exchange cross section (and, hence, the contribution of helium- $3+1$ ions) too small to be relevant in our case [13]. 
The nature of the excited ICE wave can, in principle, be determined from the available phase difference measurements (figure 3). While the presence of frequency splitting (figure 4 (a)) complicates direct wavenumber identification for core ICE, the emission takes place in the same time window as edge ICE (figure 2). This edge ICE signal appears as a pair of modes sufficiently separate in time to avoid frequency splitting (figure 5). The phase difference in this case is in the range of $0-10^{\circ}$ (figures 3 (c) and 5 (c)), where the scatter in the values is attributed to the presence of scrape-off layer turbulence [14]. The instrumentation error remains small, set by the ratio of the probe coil length $(0.02 \mathrm{~m})$ to the parallel wavelength $(1.57 \mathrm{~m}): \sim 1 \%$. The contribution of $k_{\perp}$ value is also minimal, as the probe coil tilt angle $\left(12.6^{\circ}\right)$ remains within $0.8^{\circ}$ of the local magnetic field angle $\left(11.8^{\circ}\right)$. The expected $k_{/ /}$value at the plasma region where ICE originates is small compared to $k_{\perp}$, and $k_{/ /}=0 \mathrm{~m}^{-1}$ lies within the range of our measurements.

The possibility of using ICE as an energetic ion diagnostic in D-T plasmas has been mentioned earlier [2]. Since the fusion product in this case is a (doubly-charged) alpha particle, its velocity distribution is difficult to measure directly with other diagnostics such as a neutral particle analyzer (but can be achieved using gamma-ray- and neutron-based tomography [15]). An indirect measure of fusion alphas via toroidal Alfvén eigenmode excitation may also be difficult to achieve in forthcoming D-T campaigns on JET [16]. On the other hand, previous measurements in D-T plasmas on JET [6] and TFTR [17] show that ICE is driven by fusion alphas as a collective instability with a relatively low detection threshold. This statement is reinforced by the measurements presented in our Letter since the number densities of fusion protons and tritons resulting from NBI in ASDEX Upgrade plasmas are very low. The measured ICE signal intensity and its spectral distribution across multiple harmonics depend on the extent to which the emitting energetic ion population - whether fusion-born, or created by NBI or ICRF - is super- or sub-Alfvénic with respect to the local plasma parameters and magnetic field $[18,19]$. More recent results that demonstrate a link between the energetic ion velocity distribution and ICE include measurements from JET [20] and JT-60U [21] plasmas.

To summarize, we present new measurements that support the hypothesis of a fusion product origin of core ICE on ASDEX Upgrade. The observed ICE signals have frequencies that match the fundamental hydrogen and tritium cyclotron frequencies in the plasma core (specifically, near the magnetic axis). In both cases, hydrogen and tritium are generated as a result of D-D fusion reactions between deuterium NBI ions and bulk deuterium ions. These new measurements strengthen the case for an ICE-based diagnostic to monitor the presence of fusion-born alpha particles in the core region of tritium-burning fusion plasmas in JET, ITER, CFETR, or DEMO.

This work has been carried out within the framework of the EUROfusion Consortium and has received funding from the Euratom research and training programme 2014-2018 under grant agreement No 633053. The views and opinions expressed herein do not necessarily reflect those of the European Commission. Rodolphe D'Inca's contribution to the ICE diagnostic on ASDEX Upgrade is explicitly acknowledged.

\section{References}

[1] R. Ochoukov et al., Rev. Sci. Instr. 89, 10J101 (2018).

[2] K.G. McClements, et al., Nucl. Fusion 55, 043013 (2015). 
[3] R. O. Dendy, C. N. Lashmore-Davies, and K. F. Kam, Phys. Fluids B 4, 12 (1992).

[4] M. Ichimura, et al., Nucl. Fusion 48, 035012 (2008).

[5] G.A. Cottrell, Phys. Rev. Lett. 842397 (2000).

[6] G.A. Cottrell et al., Nucl. Fusion 33, 1365 (1993).

[7] K. Thome et al. Rev. Sci. Instr. 89, 10J102 (2018).

[8] L. G. Askinazi, A. Belokurov, D. B. Gin, V. A. Kornev, S. V. Lebedev, A. E. Shevelev, A.

S. Tukachinsky and N. A. Zhubr, Nucl. Fusion 58, 082003 (2018).

[9] R. Ochoukov, V. Bobkov, H. Faugel, H. Fünfgelder, J.-M. Noterdaeme, and ASDEX

Upgrade Team, Rev. Sci. Instrum. 86, 115112 (2015).

[10] V. Mertens et al 1997 Nucl. Fusion 371607.

[11] J.G. Cordey, R.J. Goldston, and D.R. Mikkelsen, Nucl. Fusion 21, 581 (1981).

[12] R. O. Dendy, C. N. Lashmore-Davies, and K. F. Kam, Phys. Fluids B 4, 12 (1992).

[13] Summers H.P. (2004) The ADAS user manual, version 2.6. http://www.adas.ac.uk.

[14] R. Ochoukov et al. Rev. Sci. Instrum. 87, 11D301 (2016).

[15] M. Salewski et al., Nucl. Fusion 57, 056001 (2017).

[16] R.J. Dumont et al., Nucl. Fusion 58, 082005 (2018).

[17] S. Cauffman, R. Majeski, K.G. McClements and R.O. Dendy, Nucl. Fusion 35, 1597 (1995).

[18] B Reman et al, 2016 Proc. 43rd EPS Conf. on Plasma Physics (Leuven, Belgium, 4-8 July 2016) P2.041 http://ocs.ciemat.es/EPS2016PAP/pdf/P2.041.pdf

[19] R O Dendy et al, 2017 Proc. 44th EPS Conf. on Plasma Physics (Belfast, UK, 26-30 June 2017) P5.145 http://ocs.ciemat.es/EPS2017PAP/pdf/P5.145.pdf

[20] K G McClements et al, Nucl. Fusion 58, 096020 (2018).

[21] S Sumida et al, J. Phys. Soc. Japan 86, 124501 (2017). 


\section{Figures}

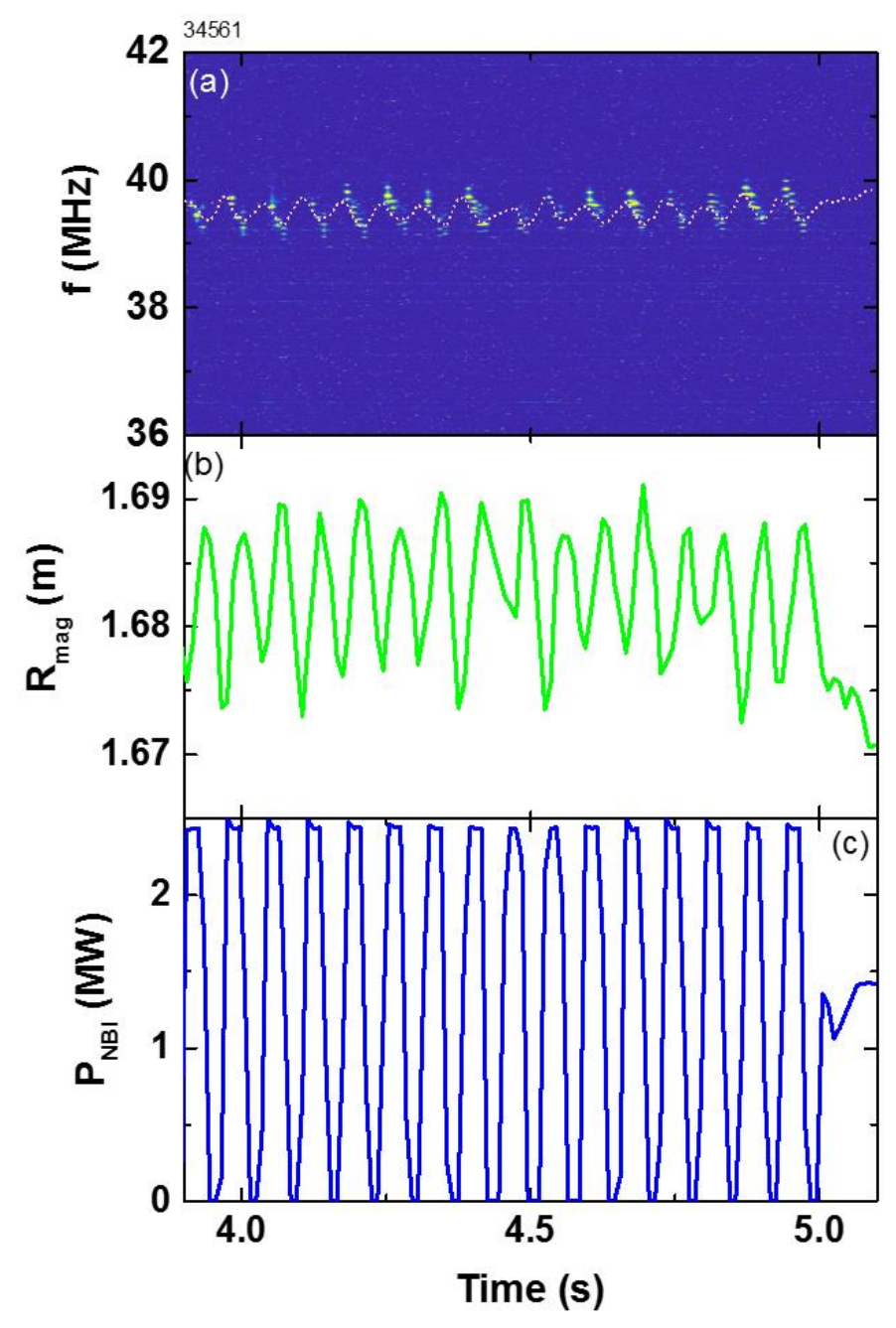

Figure 1. Example of ICE, which matches the fundamental hydrogen (and the second harmonic deuterium) cyclotron frequency in the plasma core (discharge \#34561): (a) the ICE frequency spectrum f; (b) the radial position of the magnetic axis $R_{\text {mag }}$ and (c) the neutral beam injection power $\mathrm{P}_{\mathrm{NBI}}$. The red dashed line in (a) indicates the value of the on-axis fundamental hydrogen cyclotron frequency estimated from magnetics. The on-axis magnetic field is $\mathrm{B}_{\mathrm{T}}=2.62 \mathrm{~T}$. 


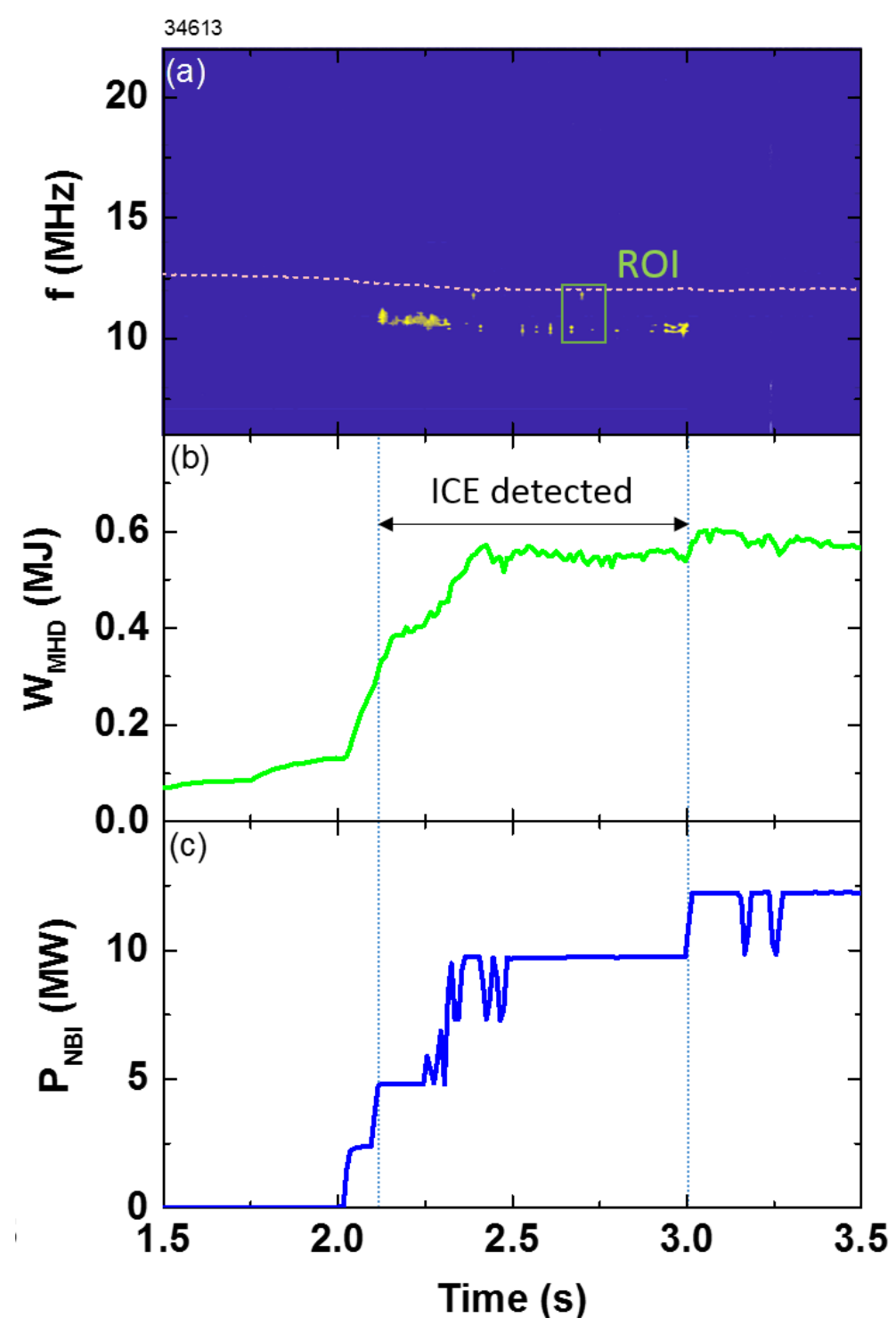

Figure 2. Example of ICE with frequencies that matches the fundamental tritium cyclotron frequency in the plasma core as well as the edge. This deuterium plasma (\#34613) is the Hmode density limit category. (a) The ICE frequency spectrum f; (b) the plasma stored energy $\mathrm{W}_{\mathrm{MHD}}$; and (c) the deuterium neutral beam power $\mathrm{P}_{\mathrm{NBI}}$ are shown. The red dashed line in (a) indicates the value of the on-axis fundamental tritium cyclotron frequency estimated from magnetics. The rectangle in (a) indicates the region of interest (ROI) plotted in figure 3 . The on-axis magnetic field is $\mathrm{B}_{\mathrm{T}}=2.37 \mathrm{~T}$ and the edge magnetic field is $\mathrm{B}=2.05 \mathrm{~T}$. 


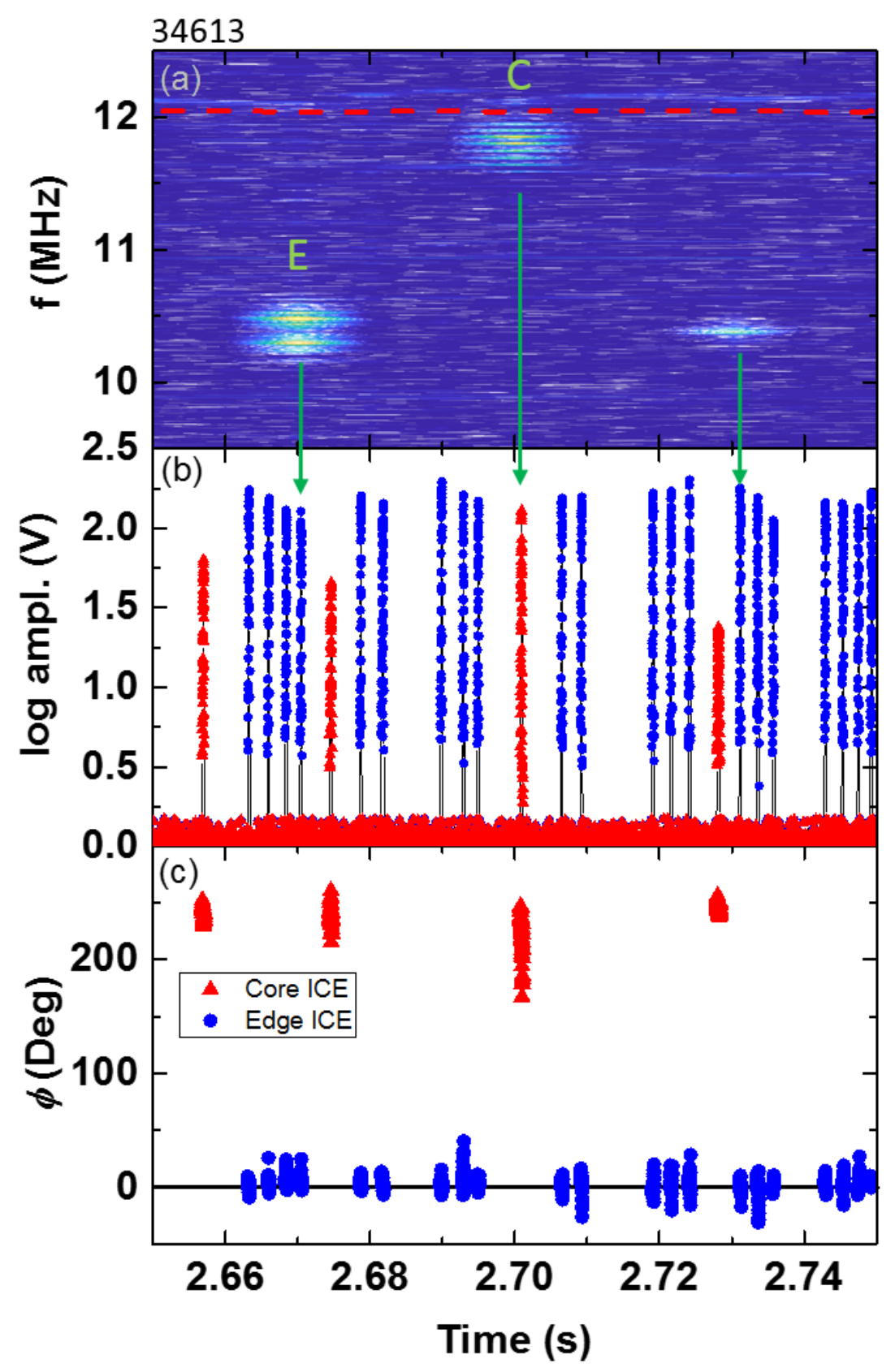

Figure 3. A detailed view of the region of interest (ROI) from figure 2 (c): (a) the ICE frequency spectrum f; (b) the ICE amplitude value; and (c) the phase difference $\phi$ between two neighboring probes. For display purpose only, each fast data acquisition burst in (a) is expanded from 1 to $10 \mathrm{~ms}$. The vertical arrows between (a) and (b) indicate the particular ICE bursts that were captured by the fast ICE diagnostic. The red dashed line in (a) indicates the value of the on-axis fundamental tritium cyclotron frequency estimated from magnetics. "C" and "E" in (a) indicate the core and the edge ICE events completely captured by the fast ICE diagnostic, see figures 4 and 5. 


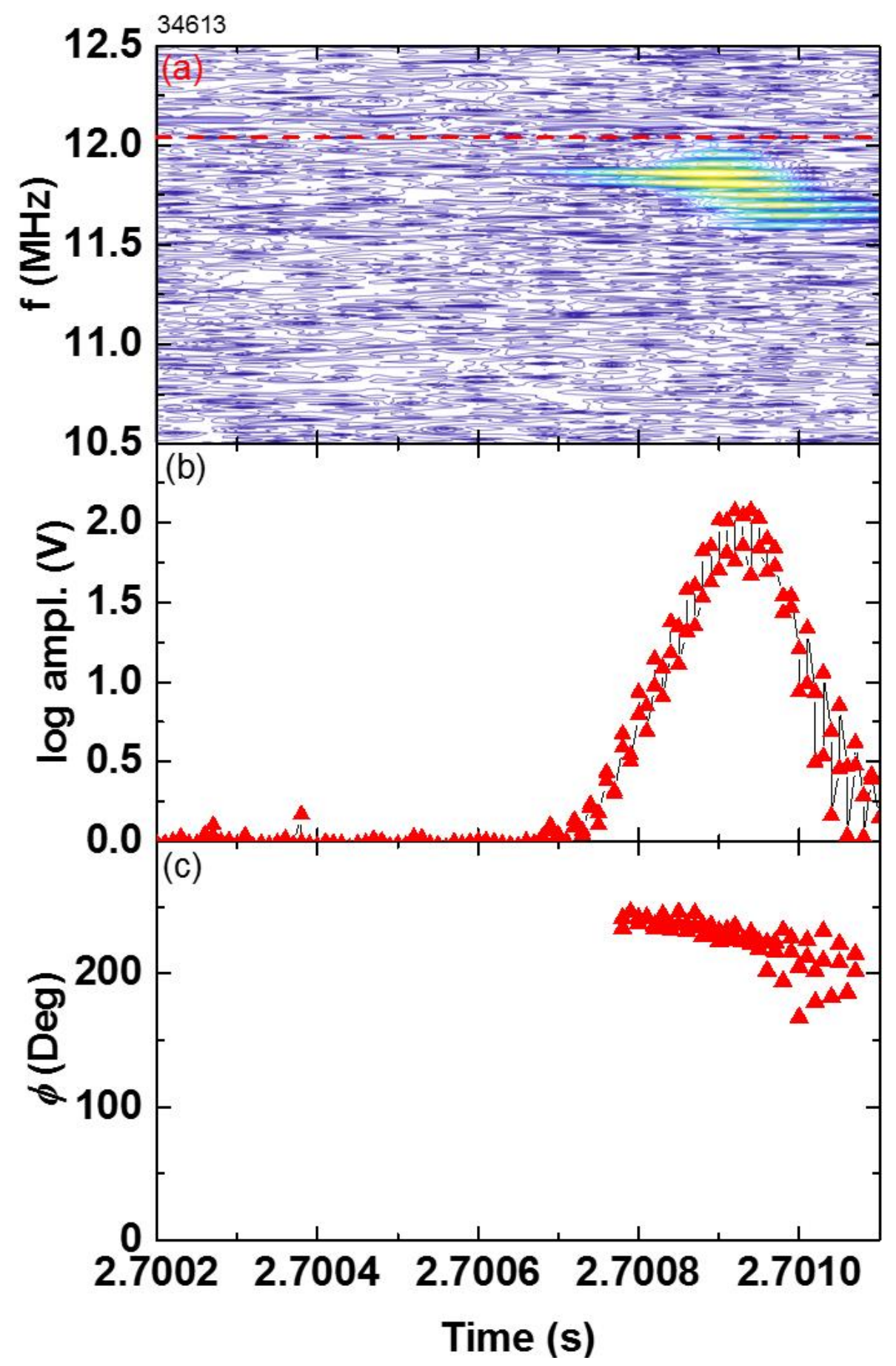

Figure 4. A detailed view of the core ICE event "C" from figure 3 (a): (a) the ICE frequency spectrum f; (b) the ICE amplitude value; and (c) the phase difference $\phi$ between the two neighboring probes is shown. The red dashed line in (a) indicates the value of the on-axis fundamental tritium cyclotron frequency estimated from magnetics. 


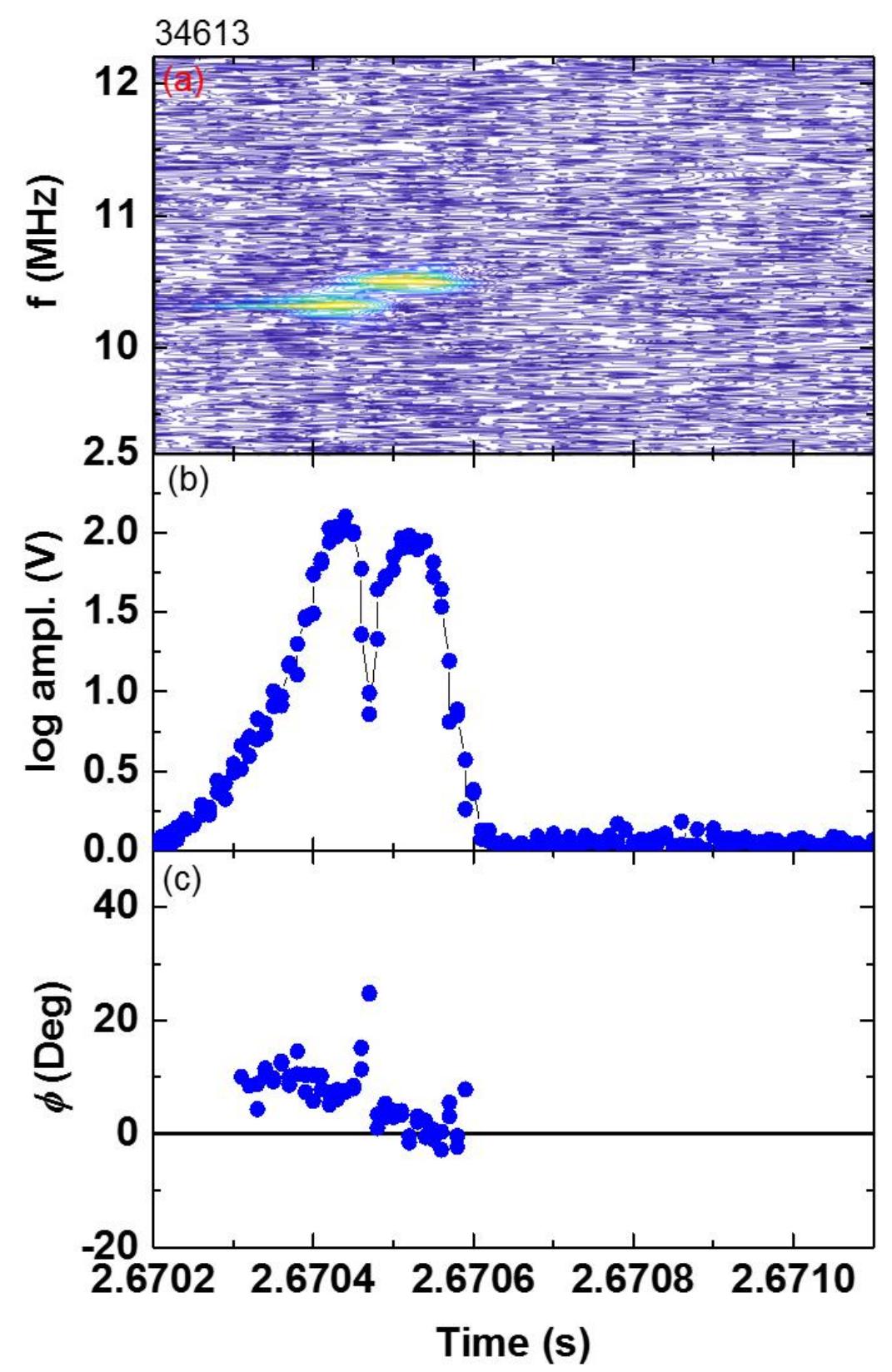

Figure 5. A detailed view of the edge ICE event "E" from figure 3 (a): (a) the ICE frequency spectrum f; (b) the ICE amplitude value; and (c) the phase difference $\phi$ between the two neighboring probes is shown. 